\title{
Effective Throughput: A Unified Benchmark for Pilot-Aided OFDM/SDMA Wireless Communication Systems
}

\author{
Dongxu Shen, Zhengang Pan, Kai-Kit Wong, Victor O.K. Li \\ Department of Electrical \& Electronic Engineering \\ The University of Hong Kong \\ Pokfulam Road, Hong Kong \\ email: \{dxshen, zgpan, kitwong, vli\}@eee.hku.hk
}

\begin{abstract}
In this paper, we study the uplink performance of an orthogonal frequency division multiplexing (OFDM) wireless system where multiple antennas are utilized at the base station (BS). Further, capacity can be greatly enhanced through spatial division multiple access (SDMA), so that several users can transmit packets simultaneously to the BS. The system performance is determined by various transmission techniques, including methods for channel estimation, modulation, as well as channel coding. Conventional parameters such as packet error rate (PER) and bit error rate (BER) are unable to reflect the actual system performance because no consideration is given to the overheads incurred by the transmission techniques. Therefore, we are motivated to propose a novel concept called $e f$ fective throughput to characterize the capacity available to users by incorporating all these factors. The effective throughput for a user can be viewed as the average number of successfully received data bits in an OFDM symbol after excluding erroneously received packets and the overheads due to channel estimation and coding. It also directly relates to the transmission delay of a user packet. The system effective throughput is the aggregated effective throughput of all users. Simulation results demonstrate that effective throughput can serve as a useful and more meaningful benchmark parameter in optimizing system performance.
\end{abstract}

Keywords - Antenna array, diversity, effective throughput, OFDM, SDMA.

\section{INTRODUCTION}

Orthogonal frequency division multiplexing (OFDM) [1], [2] is an effective method for high speed wireless communications. In OFDM, data is carried over a large number of parallel subcarriers instead of a single carrier. Thus the data rate on each subcarrier is significantly reduced, which effectively mitigates the influence of multipath fading. However, each subcarrier still undergoes flat fading that degrades system performance, especially for high level modulation. By using adaptive antenna array, the performance of OFDM can be greatly enhanced through the diversity achieved from multiple antenna elements [3]. System capacity can be further increased by spatial division multiple access (SDMA), in which more than one user can be accommodated on the same subcarrier at the same time [4], [5].

In this paper, we focus the uplink performance characterization of an OFDM/SDMA wireless communication system, where the base station (BS) is equipped with multiple receiving antennas, and multiple mobile users transmit packets to the BS through one transmitting antenna.

For data communications, data is transmitted in packets. A commonly used parameter for characterizing packet level performance is the packet error rate (PER). Given the coding method and packet length, PER is only determined by bit error rate (BER). Therefore, previous research focused on the BER performance of OFDM/SDMA systems [5], [6]. Unfortunately, PER (or BER) alone is insufficient for performance characterization; other important factors, such as the overhead from channel estimation, modulation and coding scheme, need to be included in performance analysis.

In OFDM/SDMA, there is a trade-off between PER and the overhead of channel estimation. The operation of antenna array requires the knowledge of the channel, which is often obtained through the use of pilots [7], [8]. A portion of the subcarriers is selected for pilots, and channel estimation is performed based on the received signals on pilot subcarriers [9], [10]. The quality of channel estimation improves with more pilots [11], which leads to lower BER or PER. However, since pilot subcarriers cannot be used for data, throughput is reduced. Evidently, BER (or PER) does not completely characterize the performance, so that performance optimization cannot be solely based on (the minimization of) BER. Similar trade-offs exist among PER, modulation and coding scheme. For example, high level modulation carries more bits on a subcarrier, but is prone to error; coding improves PER, but introduces redundancy. Clearly, the performance of OFDM/SDMA depends on all of the above factors that are not encompassed by PER or BER. We are thus motivated to devise a unified measure for performance characterization.

In contrast to the previous research, we define the notion of effective throughput to characterize the performance of OFDM/SDMA systems by taking into account PER, number of data subcarriers in an OFDM symbol, modulation and coding scheme. Effective throughput for a user can be viewed as the average number of successfully received data bits in an OFDM symbol after excluding erroneously received packets and the overhead of pilots as well as coding. The effective throughput for the whole system is the aggregation of effective throughput of all users. Effective throughput characterizes system performance in a more practical sense, and the optimization of system performance can be achieved through its maximization. Simulation is conducted to demonstrate the influence of various factors on effective throughput, such as the impact of pilot spacing in time and frequency, modulation and channel signal-to-noise ratio (SNR), etc.

This paper is organized as follows. In Section II, we describe the system model. In Section III, we define the effective throughput of a user and extend the idea for the system. 
We discuss how various factors affect effective throughput in Section IV. Simulation results are presented in Section V. In Section VI, we conclude this paper.

\section{SYSTEM DESCRIPTION}

In this paper, we consider the uplink (from mobile users to BS) of an OFDM system. The baseband system configuration of a mobile user for OFDM transmission is illustrated in Fig. 1. Since the operation is identical for every OFDM symbol, the time index for OFDM symbols is omitted in all later notations for conciseness. The bit stream of a user $u$ is first fed into a modulator, which maps the data bits into modulation symbols. Then a block of $N_{c}$ modulated symbols are converted from serial into parallel to produce the symbols $S_{u}(i), i=1, \ldots, N_{c}$ on the corresponding subcarriers, where $N_{c}$ is the number of subcarriers in an OFDM symbol. Next inverse fast Fourier transform (IFFT) is performed to transform the frequency domain representation $S_{u}(i), i=1, \ldots, N_{c}$ into time domain series $s_{u}(i), i=1, \ldots, N_{c}$ which are then parallel-to-serial converted. Cyclic prefix is then added to prevent inter-symbol interference (ISI). We assume that the length of the cyclic prefix is always longer than the maximal channel multipath delay spread, so that there is no ISI. Finally, the resulting signal is transmitted through a single antenna.

Assuming there are $A$ antenna elements at the $\mathrm{BS}$, and $U$ users are transmitting simultaneously to the BS. At the antenna element $a$, the received signal is first sampled at a sampling interval of $\Delta t$. Then a block of $N_{c}$ samples are converted from serial to parallel to produce $r_{a}(i), i=1, \ldots, N_{c}$, and fast Fourier transform (FFT) is performed to obtain $R_{a}(i)$, the received signal at subcarrier $i$ (see Fig. 2 for details).

The received signal $R_{a}(i)$ is the superposition of the faded versions of the signals transmitted from all users, corrupted by additive white Gaussian noise (AWGN), and can be expressed as

$$
R_{a}(i)=\sum_{u=1}^{U} H_{u, a}(i) S_{u}(i)+n_{a}(i)
$$

where $H_{u, a}(i)$ is the fading coefficient between user $u$ and the $a$ th antenna element of the BS at subcarrier $i, n_{a}(i)$ is complex AWGN, with zero mean and variance $N_{0} / 2$ per dimension.

Antenna combining is performed independently on a per subcarrier basis, and the transmitted signal for user $u$ at subcarrier $i$ can be estimated by

$$
\widehat{S}_{u}(i)=\sum_{a=1}^{A} W_{u, a}^{*}(i) R_{a}(i)
$$

where $W_{u, a}(i)$ is the complex weight at the $a$ th antenna element for user $u$, and the superscript $*$ is the complex conjugate operation.

Let $\mathbf{S}(i)=\left[S_{1}(i) S_{2}(i) \cdots S_{U}(i)\right]^{T}$ be the vector of transmitted signal on subcarrier $i$, where $T$ denotes the transpose operation. Also, let $\mathbf{R}(i)=\left[R_{1}(i) R_{2}(i) \cdots R_{A}(i)\right]^{T}$ be the received signal vector, and $\mathbf{n}(i)=\left[n_{1}(i) n_{2}(i) \cdots n_{A}(i)\right]^{T}$ be the noise vector. Then (1) can be expressed as

$$
\mathbf{R}(i)=\mathbf{H}(i) \mathbf{S}(i)+\mathbf{n}(i)
$$

where $\mathbf{H}(i)$ is the channel transfer matrix

$$
\mathbf{H}(i)=\left[\begin{array}{ccc}
H_{1,1}(i) & \cdots & H_{U, 1}(i) \\
\vdots & \ddots & \vdots \\
H_{1, A}(i) & \cdots & H_{U, A}(i)
\end{array}\right] .
$$

Denoting the antenna weight matrix as

$$
\mathbf{W}(i)=\left[\begin{array}{ccc}
W_{1,1}(i) & \cdots & W_{U, 1}(i) \\
\vdots & \ddots & \vdots \\
W_{1, A}(i) & \cdots & W_{U, A}(i)
\end{array}\right],
$$

the transmitted signal vector $\mathbf{S}(i)$ can be expressed as

$$
\widehat{\mathbf{S}}(i)=\mathbf{W}^{H}(i) \mathbf{R}(i)
$$

where $H$ represents the Hermitian transpose.

It is well-known that with channel state information, the optimal weight matrix $\mathbf{W}(i)$ can be computed by [12]

$$
\mathbf{W}(i)=\left[\mathbf{H}(i) \mathbf{H}^{H}(i)+\sigma^{2} \mathbf{I}\right]^{-1} \mathbf{H}(i)
$$

where $\mathbf{I}$ is an $A \times A$ identity matrix.

Other assumptions for the system are summarized here. The channels between any antenna pairs are uncorrelated, and signals from different users are independent. Furthermore, the number of simultaneous users is no greater than the number of antenna elements. Users are assumed to be perfectly synchronized in both time and frequency.

\section{EFFECTIVE THROUGHPUT}

\section{A. Performance Concerns}

The primary focus of this paper is on the performance characterization of an OFDM system with antenna diversity. Unlike many of the previous studies with emphasis on BER performance [3], [5], [6], we are interested in evaluating performance of the system when data is transmitted in packets.

A user packet consists of a block of bits with a certain amount of overhead, such as header and coding redundancy. A packet is protected at least by error detection coding, such as cyclic redundancy check (CRC). In this paper, we assume all bit errors can be detected by the CRC.

In packet transmission, it is desirable that a packet can be transmitted in as few OFDM symbols as possible for small transmission delay. This is equivalent to having as many data bits as possible in an OFDM symbol. On the other hand, packets should be received at an acceptable level of quality. Otherwise, erroneous packets are either retransmitted or discarded that wastes transmission efforts. To increase throughput, more bits should be put on a subcarrier; however, packet error is more likely to occur. Therefore, it is necessary to balance the number of data bits in an OFDM symbol with reception quality.

\section{B. Performance Related Factors}

Before presenting the definition of effective throughput, we identify the factors that are crucial to system performance. They are described as follows:

- Packet error rate, $P_{e}$ : PER serves as an indicator for reception quality. Whenever bit errors are detected in a packet by CRC, 


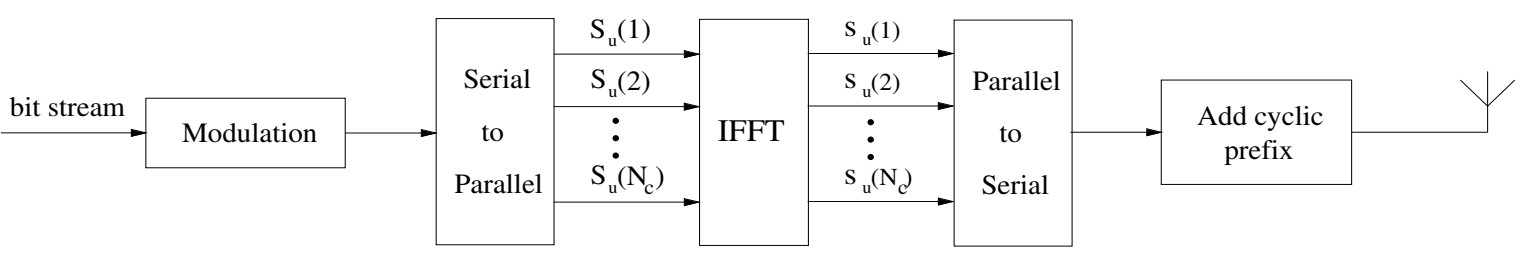

Fig. 1. System confi guration for OFDM transmission at a mobile user.

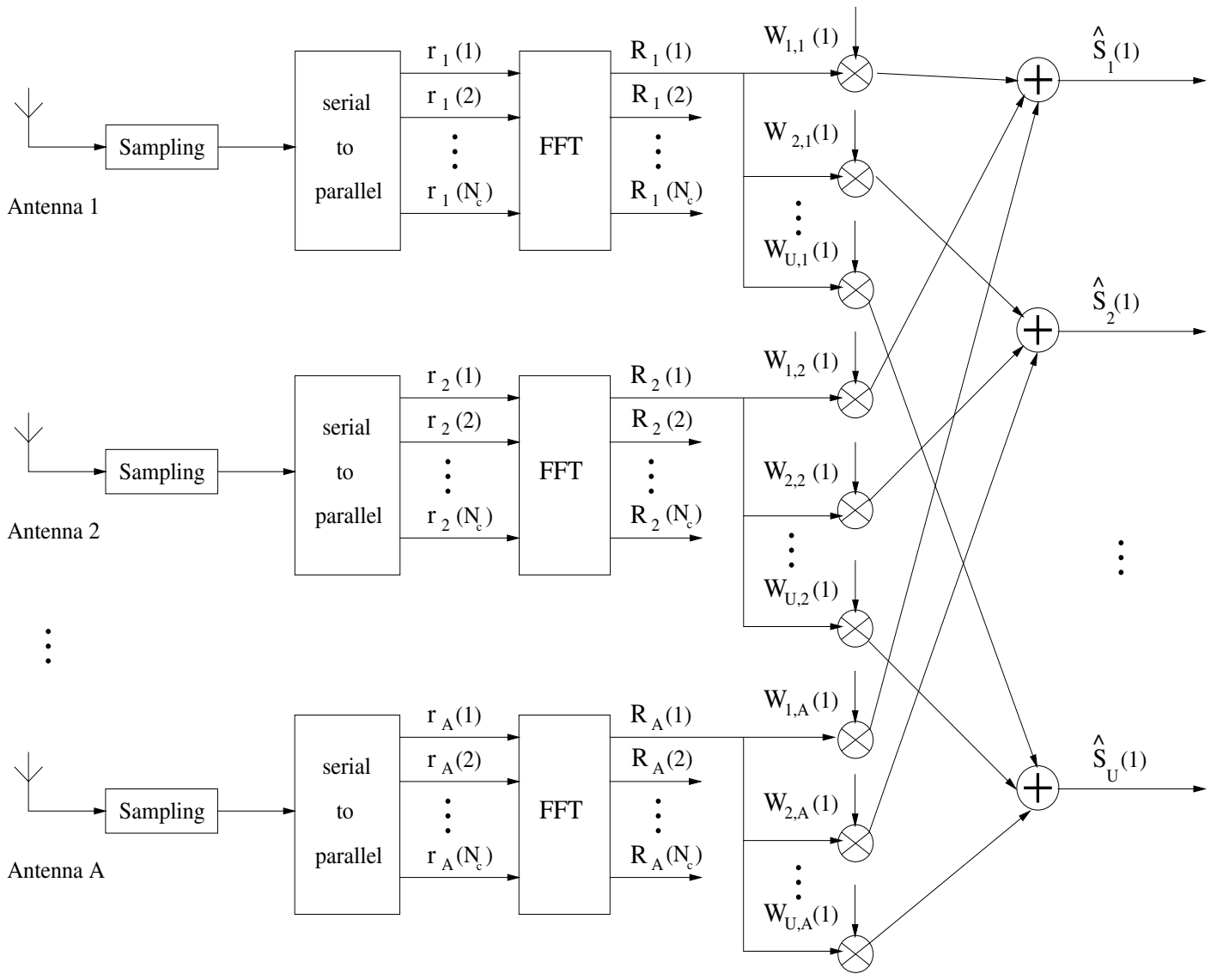

Fig. 2. System confi guration for antenna processing at the BS. The processing is identical for all subcarriers so that only subcarrier 1 is shown.

the whole packet is discarded or retransmitted. Thus, the portion of transmission efforts wasted due to bit error is represented by PER rather than BER.

- The number of data subcarriers in an OFDM symbol, $N_{d}$ : As shown in Section II, the operation of OFDM/SDMA requires the knowledge of channel information, which is usually estimated by using pilots [7], [8]. In the pilot-symbol-assisted schemes, some subcarriers are devoted as pilot subcarriers, carrying known pilot symbols, and data bits are carried by the remaining subcarriers. Therefore, the number of OFDM symbols needed to transmit a packet depends on the number of data subcarriers in an OFDM symbol.

- Modulation index, $M_{I}$ : Modulation index represents the number of bits carried on a data subcarrier. In this paper, we assume all data subcarriers employ the same modulation scheme. For a modulation scheme with a constellation size of $M_{c}$, the mod- ulation index $M_{I}$ is given by $M_{I}=\log _{2} M_{c}$, which gives the number of bits on a modulated symbol. Although higher order modulation carries more bits on one data subcarrier, it is more susceptible to error, which may offset the gain by having more bits in a subcarrier. For example, QPSK (quadrature phase shift keying), 16 QAM (quadrature amplitude modulation) and 64 QAM carry 2, 4 and 6 bits/symbol respectively, but BER performance deteriorates from QPSK to 64 QAM with the same per bit energy [11].

- Coding rate, $r_{c}$ : The level of redundancy due to channel coding can be characterized by the coding rate

$$
r_{c}=\frac{R_{d}}{R_{c}}
$$

where $R_{d}$ is the number of data bits, and $R_{c}$ is the number of coded bits. 


\section{Definition of Effective Throughput}

The concept of effective throughput aims to integrate the above factors to provide a unified performance measure for pilot-assisted OFDM/SDMA wireless communication systems. Definition 1-User Effective Throughput: Let $P_{e}$ be the PER, $N_{d}$ the number of data subcarriers in an OFDM symbol, $M_{I}$ the modulation index on data subcarriers, and $r_{c}$ the coding rate. The effective throughput of a user is defined as

$$
T_{u} \triangleq\left(1-P_{e}\right) N_{d} M_{I} r_{c}
$$

The normalized effective throughput is defined as the ratio between the user effective throughput and the number of subcarriers in an OFDM symbol. That is

$$
\begin{aligned}
\bar{T}_{u} & \triangleq \frac{T_{u}}{N_{c}} \\
& =\frac{\left(1-P_{e}\right) N_{d} M_{I} r_{c}}{N_{c}} .
\end{aligned}
$$

Definition 2-System Effective Throughput: Let $T_{u}$ be the effective throughput for user $u$. When there are $U$ users transmitting simultaneously, the system effective throughput is defined as

$$
T_{a} \triangleq \sum_{i=1}^{U} T_{u} .
$$

The normalized system effective throughput is similarly defined as

$$
\begin{aligned}
\bar{T}_{a} & \triangleq \sum_{i=1}^{U} \bar{T}_{u} \\
& =\frac{T_{a}}{N_{c}} .
\end{aligned}
$$

The definition of effective throughput considers the influence of PER $\left(P_{e}\right)$, the overhead introduced by pilots $\left(N_{d}\right)$, modulation index $\left(M_{I}\right)$, and coding redundancy $\left(r_{c}\right)$. It incorporates both packet reception quality and the number of data bits in an OFDM symbol. The reception quality is represented by $\left(1-P_{e}\right)$, which is the portion of packets correctly received. In an OFDM symbol, only $N_{d}$ out of $N_{c}$ subcarriers contribute to carrying user data. On each data subcarrier, the number of data bits is $M_{I}$. Also, at a coding rate $r_{c}$, the number of (precoded) bits in an OFDM symbol is $N_{d} M_{I} r_{c}$. Thus the effective throughput $\left(1-P_{e}\right) N_{d} M_{I} r_{c}$ represents the average number of bits that is available for transmitting packets in an OFDM symbol. Correspondingly, the normalized effective throughput gives the average number of data bits on a subcarrier.

The effective throughput can be easily translated to the average delay for transmitting a user packet when retransmission is employed. Let $L_{d}$ be the packet size before channel coding, or $L_{d} / r_{c}$ after channel coding. The number of OFDM symbols needed to transmit a packet is

$$
\frac{L_{d} / r_{c}}{N_{d} M_{I}}
$$

which corresponds to a period of

$$
\left(\frac{L_{d} / r_{c}}{N_{d} M_{I}}\right) T_{S}
$$

where $T_{S}$ is the duration of an OFDM symbol, and is a fixed parameter.

Assuming that an erroneous packet is always retransmitted until successfully received, and the occurrence of a packet error is independent, identically distributed (i.i.d.). Then the number of transmission attempts is a geometric random variable and the average number of transmission attempts is given by

$$
\frac{1}{1-P_{e}}
$$

Accordingly the average delay $\tau_{D}$ for transmitting a data packet is

$$
\begin{aligned}
\tau_{D} & =\frac{L_{d}}{\left(1-P_{e}\right) N_{d} M_{I} r_{c}} \times T_{S} \\
& =\left(\frac{L_{d}}{T_{u}}\right) T_{S} .
\end{aligned}
$$

Since $T_{S}$ is a fixed value, at a given packet length, the average delay is solely determined by the effective throughput $T_{u}$. Consequently, minimizing the average packet delay is equivalent to maximizing the effective throughput.

\section{Discussions}

Effective throughput is determined by PER, the number of data subcarriers (or the number of pilot subcarriers), modulation and coding scheme. In this section, we discuss the influence of these factors on effective throughput.

\section{A. Packet Error Rate}

By definition, effective throughput is proportional to $\left(1-P_{e}\right)$. PER relates to BER as

$$
P_{e}=1-\left(1-P_{b}\right)^{L_{d}},
$$

where $P_{b}$ is BER, and $L_{d}$ is the length of a packet. When $L_{d} P_{b} \ll 1$,

$$
P_{e} \approx L_{d} P_{b} .
$$

Thus if the packet length is fixed, $P_{e}$ is determined by $P_{b}$.

In OFDM, $P_{b}$ is influenced by channel SNR, quality of channel estimation, modulation and coding scheme. With antenna array, it is also affected by the number of antenna elements and the number of simultaneous users. From the system viewpoint, the number of antenna elements at the BS is fixed. Therefore, $P_{b}$ is influenced by the number of simultaneous users $U$. It is known [12] that an $A$-element array has $A-1$ degrees of freedom. The cancellation of CCI for one user costs one degree of freedom. When there are $U$ users, $U-1$ degrees of freedom are dedicated for CCI cancellation, and the remaining $A-U$ degrees of freedom are for diversity. Apparently, the level of diversity decreases with more users. With less diversity, $P_{b}$ and correspondingly $P_{e}$ increase. This means the effective throughput for a user is always decreasing with more users. However, system effective throughput $T_{a}$ may still increase. 


\section{B. Pilot Subcarriers}

In this paper, channel estimation is obtained through pilots. The number of pilots determines the quality of channel estimation, and hence the PER performance. However, the use of pilots causes the reduction of effective throughput. With SDMA, we show in this subsection that the overhead from pilots cannot be neglected.

\section{B.1 Arrangement of Pilot Subcarriers}

In OFDM/SDMA systems, channel estimation requires the proper arrangement of pilots. For a user $u$, let $S_{u}(p)$ be the pilot symbol on pilot subcarrier $p, R_{a}(p)$ be the received signal at antenna $a$. Then $R_{a}(p)$ is expressed as

$$
R_{a}(p)=H_{u, a}(p) S_{u}(p)+I(p)+n(p),
$$

where $H_{u, a}(p)$ is the channel response on subcarrier $p$ between user $u$ and receiving antenna $a, I(p)$ is the CCI from other users, and $n(p)$ is AWGN. The channel response $H_{u, a}(p)$ can be estimated by

$$
\widehat{H}_{u, a}(p)=\frac{R_{a}(p)}{S_{u}(p)} .
$$

When $I(p)=0$ (i.e., no CCI), the quality of channel estimation from (17) only depends on the SNR on subcarrier $p$.

If another user $u^{\prime}$ transmits data on a pilot subcarrier of user $u$, CCI from user $u^{\prime}$ can severely degrade the quality of channel estimation. Therefore, CCI should be avoided on all pilot subcarriers, which calls for the proper arrangement of pilots among all users.

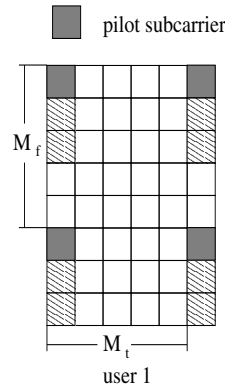

ser 1

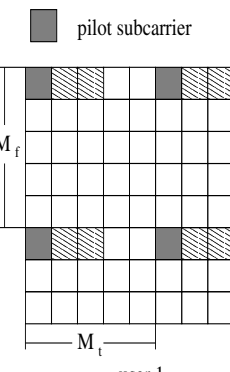

user 1

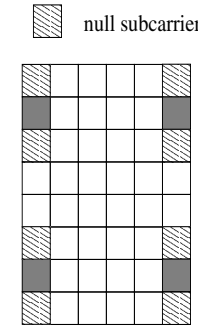

user 2

(a)

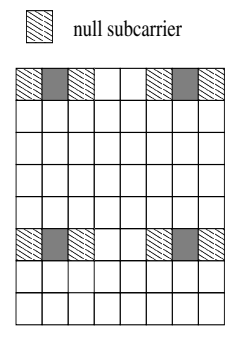

user 2 data subcarrier

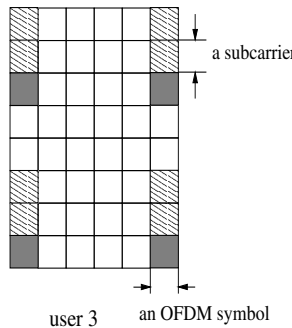

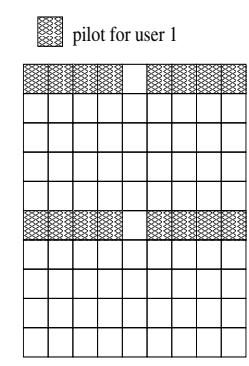

user 1
To this end, one solution is to ensure the pilot subcarriers used by one user are not used as data subcarriers for all other users. As plotted in Fig. 3, when a subcarrier is used by a user as a pilot subcarrier, other users put a null symbol on the subcarrier (i.e., no symbol is carried, neither pilot nor data). We refer to this approach as the null subcarrier (NS) method.

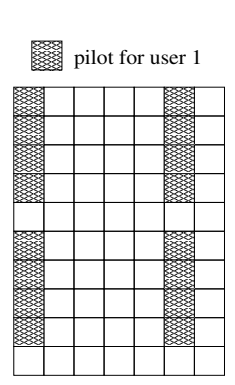

user 1

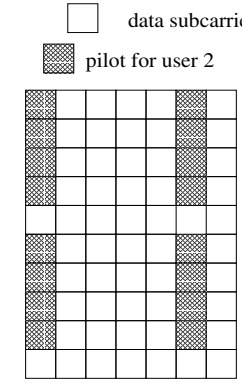

user 2

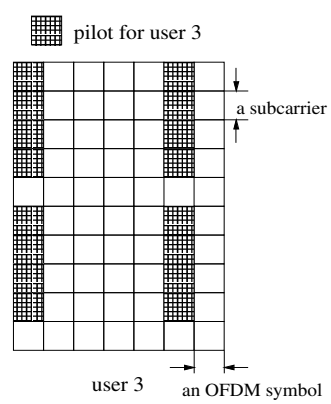

(a)

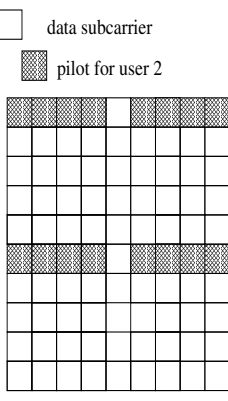

user 2

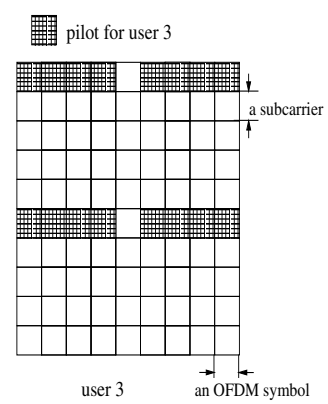

(b)
Fig. 4. Pilot arrangement using orthogonal spreading sequences: (a) spreading across subcarriers in the same OFDM symbol; (b) spreading across symbols at the same subcarrier.

In [6], two other pilot arrangement methods were proposed, both based on orthogonal spreading sequences. In one scheme, as shown in Fig. 4 (a), a Walsh spreading sequence is used to spread a pilot onto multiple sequential subcarriers. Users are assigned with different spreading sequences, while pilots are spread onto the same group of subcarriers. Due to the orthogonality among Walsh sequences, no CCI is introduced under perfect synchronization. We call this method frequency-domain spreading (FS) scheme. Another scheme is to spread a pilot on the same subcarrier of successive OFDM symbols by Walsh sequences, as illustrated in Fig. 4 (b). We call this time-domain spreading (TS) scheme.

All of the above schemes require a 2-D channel estimation [10], [13] to be performed based on the channel gains obtained at the pilot locations (using (17)).

\section{B.2 Comparison of Pilot Arrangement Schemes}

Pilot subcarriers are not available for data, and are thus overheads. Among the three pilot arrangement schemes introduced previously, NS is more favorable than the other two, because it has less overhead, and exhibits potential for better channel esti- 
mation.

For NS, the overhead consists of the pilot subcarriers and null subcarriers. When there are $U$ users, each pilot requires one pilot subcarrier and $U-1$ null subcarriers, which corresponds to $U$ subcarriers of overhead for one pilot.

We can show that the overhead of FS and TS is no smaller than that of NS. Since the length of a Walsh sequence is a power of 2 , the number of subcarriers for one pilot in the FS and TS schemes is

$$
L_{s}=2^{\left\lceil\log _{2} U\right\rceil}
$$

where $U$ is the number of users, and $\lceil x\rceil$ denotes the smallest integer that is larger than or equal to $x$. Obviously, $L_{s} \geq U$. Thus the overhead for FS and TS schemes is always no smaller than that of NS. In some cases, the difference can be significant. For example, when $U=5$, NS needs 5 subcarriers for one pilot, while FS and TS need 8 subcarriers, which means $60 \%$ more overhead than NS.

On the other hand, FS and TS implicitly assume that the channel gain remains constant across the subcarriers or symbols. However, channel response varies in both frequency due to the channel delay spread and time due to the Doppler spread. Therefore, the estimation from the two schemes would be less accurate than that from NS.

\section{B.3 Overhead of Pilots}

In OFDM/SDMA, the effective throughput reduction caused by pilots could be significant. We illustrate this by the following example.

Example 1: OFDM is adopted in wireless local area networks (LAN) standards such as IEEE 802.11a [14] and HIPERLAN II [15]. In these systems, antenna array is not used at the BS. Only one user can communicate with the BS at one time. Pilots are carried in every OFDM symbol. In each OFDM symbol, there are 4 pilot subcarriers and 48 data subcarriers out of 64 subcarriers (there are 12 null subcarriers for spectrum shaping). This represents an overhead of $4 / 52 \approx 8 \%$.

In contrast, for an OFDM/SDMA system, suppose that there are 6 antennas at the BS to support 5 users. Using the NS method and assuming again 4 pilots in each OFDM symbol, we need $4 \times$ $5=20$ subcarriers for pilots and null subcarriers for each user. This means $20 / 52 \approx 40 \%$ of overhead in an OFDM symbol.

This example reveals that overhead from pilots can significantly reduce effective throughput in the presence of multiple simultaneous users. The implication is that we can hardly afford to have pilots in every OFDM symbol, and pilots should be dispersed in time. As such, 2-D channel estimation [10], [13] is required. The gain due to the reduction of pilot density is obvious, and is shown by the next example.

Example 2: If pilots are inserted once in every 5 OFDM symbols, the overhead is reduced by 5 times to $8 \%$ as compared to $40 \%$ in Example 1.

In the definitions of effective throughput, $N_{d}$ is the average number of data subcarriers in an OFDM symbol. Suppose that pilots are arranged in such a way that there are $N_{P}$ subcarriers for pilots (including null subcarriers) in every block of $B_{s}$ OFDM symbols. Then $N_{d}$ is calculated as

$$
N_{d}=N_{c}-N_{P} / B_{s}
$$

\section{B.4 Optimizing the Number of Pilots}

Reducing the number of pilot subcarriers can increase the number of data subcarriers. However, channel estimation is less accurate with fewer pilots, which leads to higher BER and PER. Thus minimizing pilots should be carefully balanced with PER performance.

The definition of effective throughput provides the criterion for optimizing the number of pilot subcarriers. Since effective throughput is proportional to

$$
\left(1-P_{e}\right) N_{d}
$$

obviously the best selection of pilot density is to maximize the product in (20).

This also reveals that effective throughput is a better parameter for system performance than PER or BER. It can automatically balance between PER and pilot density.

\section{Modulation Scheme}

Similarly, the selection of modulation scheme should maximize

$$
\left(1-P_{e}\right) M_{I}
$$

For example, we compare two modulation schemes, QPSK $\left(M_{I}=2\right)$ and 16 QAM $\left(M_{I}=4\right)$. Let $P_{e, 4}$ and $P_{e, 16}$ be the corresponding PER. Then 16 QAM is more preferable than QPSK only when

$$
\left(1-P_{e, 4}\right)<2\left(1-P_{e, 16}\right),
$$

or

$$
P_{e, 16}<\frac{1+P_{e, 4}}{2}
$$

Without antenna array, the performance of various modulation schemes for OFDM mainly depends on SNR and channel conditions [11], [16]. With antenna array, the performance is further related to the level of antenna diversity, and the quality of channel estimation.

\section{Channel Coding}

The optimal coding scheme should be selected by maximizing

$$
\left(1-P_{e}\right) r_{c} \text {. }
$$

The influence of channel coding on OFDM has been investigated in [17], [18] without antenna diversity, and in [19], [20] with antenna diversity. The impact of coding on effective throughput is a non-trivial issue. In this paper, we do not consider the use of coding. A thorough investigation of this topic requires further study in the future.

\section{Simulation Results}

In this section, we present the simulation results for an OFDM/SDMA system. The influence of various factors on BER and effective throughput performance is demonstrated. 


\section{A. Simulation Parameters}

The parameters of the simulation are summarized in Table I. The coding rate $r_{c}=1$ because channel coding is not employed.

TABLE I

PARAMETERS FOR AN OFDM/SDMA SYSTEM.

\begin{tabular}{|c|c|}
\hline Number of antennas & $A=6$ \\
\hline Carrier frequency & $f_{c}=5.4 \mathrm{GHz}$ \\
\hline Bandwidth & $20 \mathrm{MHz}$ \\
\hline Number of subcarriers & $N_{c}=128$ \\
\hline Carrier spacing & $\Delta f=20 / 128=162.5 \mathrm{kHz}$ \\
\hline Sampling interval & $\Delta t=1 / N \Delta f=0.05 \mu \mathrm{s}$ \\
\hline FFT period & $T_{F}=1 / \Delta f=6.4 \mu \mathrm{s}$ \\
\hline Guard interval & $T_{G}=0.8 \mu \mathrm{s}$ \\
\hline Symbol interval & $T_{S}=T_{F}+T_{G}=7.2 \mu \mathrm{s}$ \\
\hline Packet length & $L_{d}=500 \mathrm{bytes}$ \\
\hline Modulation & $\mathrm{QPSK}, 16 \mathrm{QAM}, 64 \mathrm{QAM}$ \\
\hline Coding rate & $r_{c}=1$ \\
\hline Pilot SNR & $20 \mathrm{~dB}$ \\
\hline
\end{tabular}

We assume all users apply the same operating parameters so that they have the same pilot pattern and modulation scheme. The channels between every user and the BS are statistically identical and independent. Therefore, all users should have statistically the same BER and PER performance. In what follows, the effective throughput of the system is equivalent to the effective throughput of a user multiplied by the number of users. Thus we only provide the results of user effective throughput.

\section{B. Channel Simulation and Estimation}

We assume the multipath fading channel is wide sense stationary with uncorrelated scattering (WSSUS). The channel impulse response $h(\tau)$ is modeled as a tapped delay line with tap spacing $\Delta t$, and is written as

$$
h(\tau)=\sum_{l=0}^{L_{p}} h_{l} \delta(\tau-l \Delta t),
$$

where $L_{p}$ is the number of paths, $h_{l}$ is the complex fading coefficient at path $l, \delta(\cdot)$ is the Dirac delta function.

$L_{p}$ is called the channel length and is determined from the maximal delay spread $\tau_{\max }$ by

$$
L_{p}=\left\lceil\frac{\tau_{\max }}{\Delta t}\right\rceil \text {. }
$$

We also assume that the maximal delay spread is always smaller than the guard interval. From our simulation parameters, this means

$$
L_{p} \leq \frac{T_{G}}{\Delta t}=16
$$

Expressing the fading coefficient as $h_{l}=A_{l} e^{j \theta_{l}}$, we model the fading amplitude $A_{l}$ by a Rayleigh distributed random variable (r.v.), and the phase $\theta_{l}$ by a uniformly distributed r.v. between 0 and $2 \pi$. In addition, the average power of $h_{l}$ follows an exponential decay profile

$$
E\left\{A_{l}^{2}\right\}=A_{0} e^{-\frac{l \Delta t}{\tau_{r m s}}}
$$

where $\tau_{r m s}$ is the root mean square (rms) delay spread, and $A_{0}$ is a power coefficient. In theory, $\tau_{\max }$ can be infinitely large. From practical considerations, we limit $\tau_{\max }=5 \times \tau_{r m s}$ for convenience, because it is easy to calculate that the power beyond $5 \times \tau_{r m s}$ is less than $1 \%$ of the total power. In the simulation, we use $L_{p}$ as a parameter for channel delay spread instead of $\tau_{r m s}$ or $\tau_{\max }$. Since we also require $\frac{\tau_{\max }}{\Delta t}$ to be an integer, (27) can be rewritten as

$$
E\left\{A_{l}^{2}\right\}=A_{0} e^{-\frac{5 l}{L_{p}}} .
$$

The Doppler frequency $F_{d}$ is normalized with respect to the period of an OFDM symbol $T_{S}$ as $F_{d} T_{S}$. In our simulation, $F_{d} T_{S}$ is between 0.001 and 0.01 . At $T_{S}=7.2 \mu \mathrm{s}$, these values correspond to $F_{d}$ between $138.9 \mathrm{~Hz}$ and $1389 \mathrm{~Hz}$, which are equivalent to vehicular speeds of $7.72 \mathrm{~m} / \mathrm{s}(27.8 \mathrm{~km} / \mathrm{h})$ and $77.2 \mathrm{~m} / \mathrm{s}(277.8 \mathrm{~km} / \mathrm{h})$ at the carrier frequency of $5.4 \mathrm{GHz}$. Those values are high enough for our interests. In our simulation, we adopt the modified Jakes method in [21] to generate Rayleigh faded channel traces.

Channel estimation is achieved through pilots. The NS method illustrated in Section IV-B.1 is adopted for pilot arrangement, in which pilots are placed on rectangular grid of subcarriers [13]. The separation of pilots is $M_{f}$ across subcarriers, and $M_{t}$ across OFDM symbols, as illustrated in Fig. 3 (b). Maximal likelihood (ML) estimation [9] is first performed within the subcarriers of an OFDM symbol. After applying ML estimation on two pilot-inserted OFDM symbols (which are $M_{t}$ symbols apart), linear interpolation is used to obtain channel estimation for the symbols in between [10]. The ML method involves matrix inversion. To have an invertible matrix, the ML algorithm requires the number of pilots in an OFDM symbol to be greater than the channel length, i.e., $\frac{N_{c}}{M_{f}} \geq L_{p}$. Since we confine $L_{p} \leq 16, M_{f} \leq 8$ is sufficient for all values of $L_{p}$ when $N_{c}=128$. In our simulation, the channel SNR on the pilot subcarriers is always maintained at $20 \mathrm{~dB}$ for reliable channel estimation regardless of the SNR on data subcarriers.

\section{Influence of Channel Conditions}

We first present simulation results for various channel conditions. The modulation scheme is QPSK with received SNR $\frac{E_{b}}{N_{0}}=20 \mathrm{~dB}$, where $E_{b}$ is the per bit energy on an antenna element, and $N_{0}$ is noise power. We also set $M_{f}=8$ and $M_{t}=5$. The number of users is fixed at $U=3$.

In Fig. 5, BER results under various channel conditions are provided. It can be seen that $F_{d} T_{S}$ has little influence on performance. This shows that $M_{t}=5$ is sufficient for accurate channel estimation for all $F_{d} T_{S}$ values under investigation. The BER is around $10^{-6}$ when $L_{p} \leq 12$. However, when $L_{p}=16$, BER degrades to $10^{-5}$. This indicates channel length has a more profound effect on BER performance than normalized Doppler frequency for our simulation parameters.

In Fig. 6, we present results on the normalized effective throughput. As defined in (10), the normalized effective throughput has a unit in the number bits per subcarrier. In the subsequent discussions, the effective throughput and the nor- 


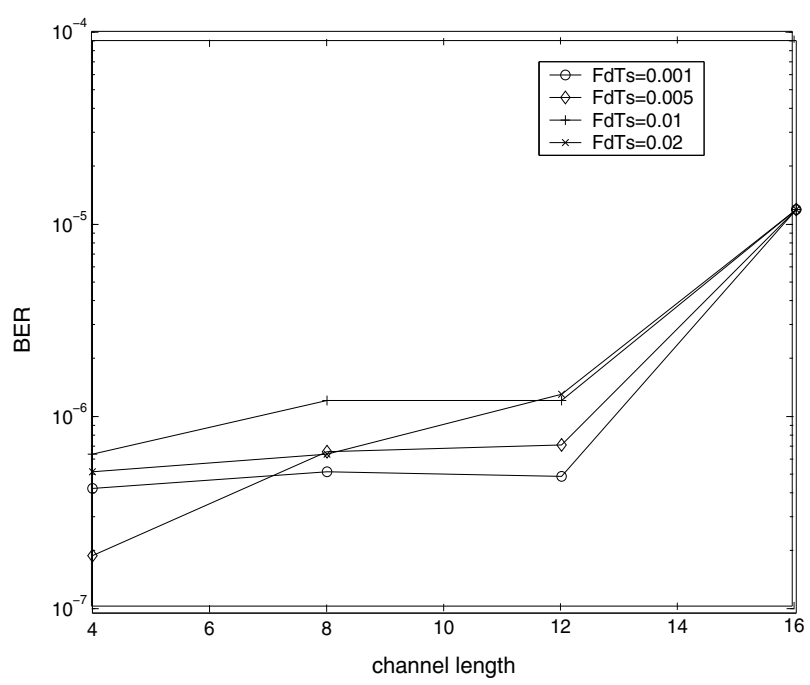

Fig. 5. BER under various channel conditions, $M_{f}=8, M_{t}=5, U=3$.

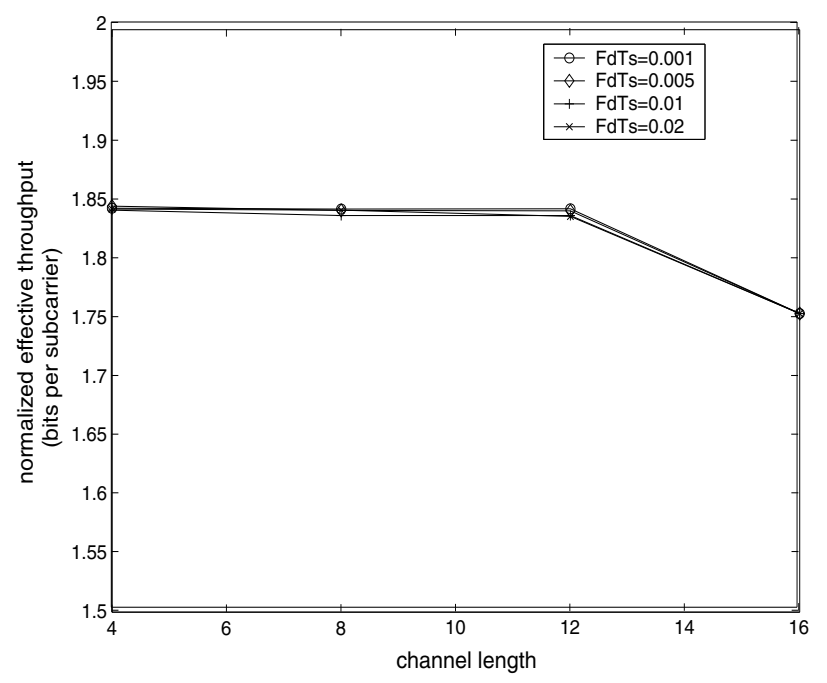

Fig. 6. Normalized effective throughput under various channel conditions, $M_{f}=8, M_{t}=5, U=3$.

malized effective throughput are used interchangeably for simplicity.

Note from Fig. 6 that the effective throughput is similar for different values of $F_{d} T_{S}$. When $L_{p} \leq 12$, effective throughput is around 1.85 bits/subcarrier, which is close to the theoretical limit of 2 bits/subcarrier for QPSK. It can be calculated from (19) that $N_{d} / N_{c}=0.925$, which corresponds to 1.85 bits/subcarrier if there is no PER. This implies that the difference ( 0.15 bits/subcarrier) is mainly caused by the overhead of pilots. When $L_{p}=16$, effective throughput is about 1.76 bits/subcarrier. Although the BER for $L_{p}=16$ is higher than those for $L_{p} \leq 12$ by an order, the corresponding effective throughput is lower by only 0.08 bits/subcarrier. Consequently, for QPSK with $M_{t}=5$ and $M_{f}=8$, effective throughput is very close to the maximal allowable level for all channel conditions.
Given our simulation settings, the results reveal that the channel length is more influential on performance than Doppler spread. With a moderate $M_{t}$, for example $M_{t}=5$, variation in Doppler spread hardly impacts performance. On the other hand, long channel length tends to degrade performance. For this reason, we focus our attention on the worst case of channel conditions with $L_{p}=16$ and $F_{d} T_{S}=0.01$.

\section{Influence of Pilot Spacing}

In this subsection, we demonstrate the influence of pilot spacing on the performance for various modulation schemes, including QPSK, 16 QAM and 64 QAM. In the simulation, we fix $L_{p}=16, F_{d} T_{S}=0.01$, as pointed out previously. The number of users is 3 . To have a fair comparison between different modulation schemes, we require all modulation schemes to have the same $E_{b} / N_{0}=20 \mathrm{~dB}$ on a data subcarrier.

In addition, we use $\left(M_{f}, M_{t}\right)$ to denote a selection of pilot spacing in frequency and time. With $\left(M_{f}=4, M_{t}=20\right)$ (or $M_{f}=8, M_{t}=10$ ), the overhead for pilots is only $3.75 \%$ of total subcarriers when there are 3 users (see Section IV-B.3). With $\left(M_{f}=4, M_{t}=10\right)$ (or $\left.M_{f}=8, M=5\right)$ ) and $\left(M_{f}=\right.$ $\left.4, M_{t}=5\right)$, the overheads are respectively $7.5 \%$ and $15 \%$.

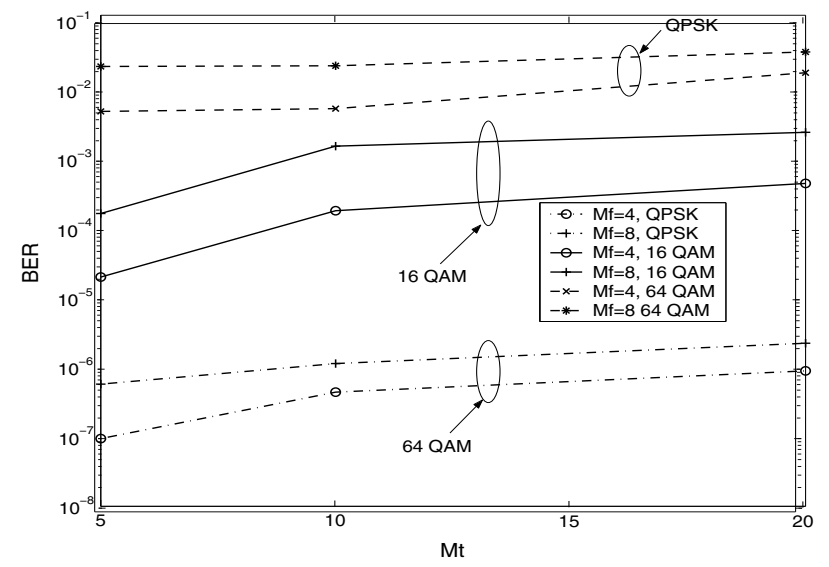

Fig. 7. BER for QPSK, 16 QAM and 64 QAM with different pilot spacing.

In Fig. 7, we plot the BER performance with different values of $M_{t}$ and $M_{f}$. Given the same $M_{t}$, the cases with $M_{f}=4$ always outperform those with $M_{f}=8$. This is natural since more pilots are used with $M_{f}=4$ than $M_{f}=8$. Interestingly, for the pilot spacings with the same amount of overhead (in this example, pilot spacings of $\left(M_{f}=4, M_{t}=10\right)$ versus $\left(M_{f}=\right.$ $\left.8, M_{t}=5\right)$ and $\left(M_{f}=4, M_{t}=20\right)$ versus $\left(M_{f}=8, M_{t}=\right.$ 10) have the same overheads), the BER performance all favors the combinations with $M_{f}=4$. This shows pilot spacing in the frequency domain is more critical than in the time domain.

The results for effective throughput are shown in Fig. 8. We notice that the effective throughput for QPSK increases with fewer pilots, but the improvement is trivially small. The reason is that the increase in PER offsets the reduction in pilot overhead. It is also clear from Fig. 8 that $M_{t}=10$ and $M_{t}=20$ are not viable for 16 QAM and 64 QAM, since they can lead to zero effective throughput. Therefore, in later simulations, we always 
set $M_{t}=5$.

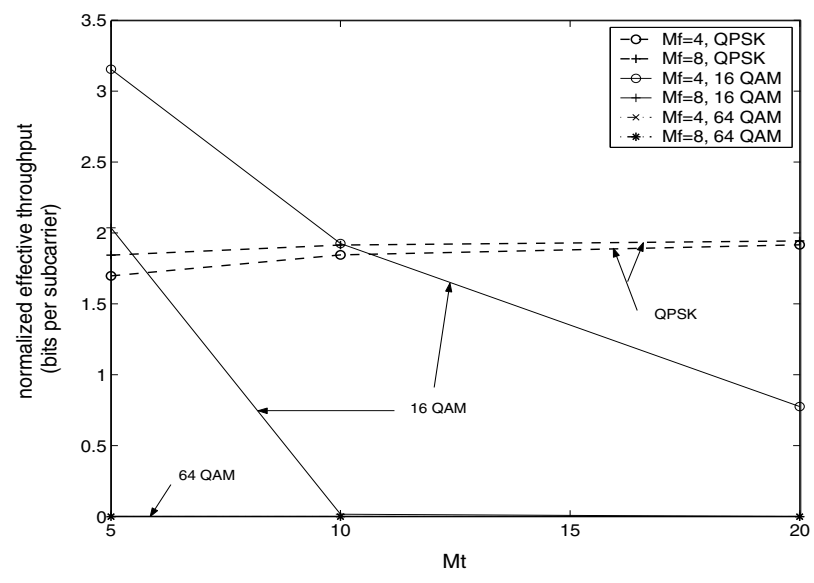

Fig. 8. Normalized effective throughput for 16 QAM and 64 QAM under different pilot spacing.

From Fig. 8, we also observe that 16 QAM achieves the highest effective throughput of 3.2 bits/subcarrier at $\left(M_{f}=\right.$ $4, M_{t}=5$ ) among all experimental results. It is interesting to note that although the BER performance of QPSK is much better than that of 16 QAM, higher effective throughput is achieved with 16 QAM when $M_{t}=5$. This observation confirms our previous claim that BER (or PER) alone is insufficient to characterize system performance.

\section{E. Number of Users}

We present results on how the system performance is affected by the number of users. QPSK is used with $E_{b} / N_{0}=20 \mathrm{~dB}$ and pilot spacing $\left(M_{f}=8, M_{t}=5\right)$. The Doppler spread is set as $F_{d} T_{S}=0.01$, and channel length varies from 4 to 16 .

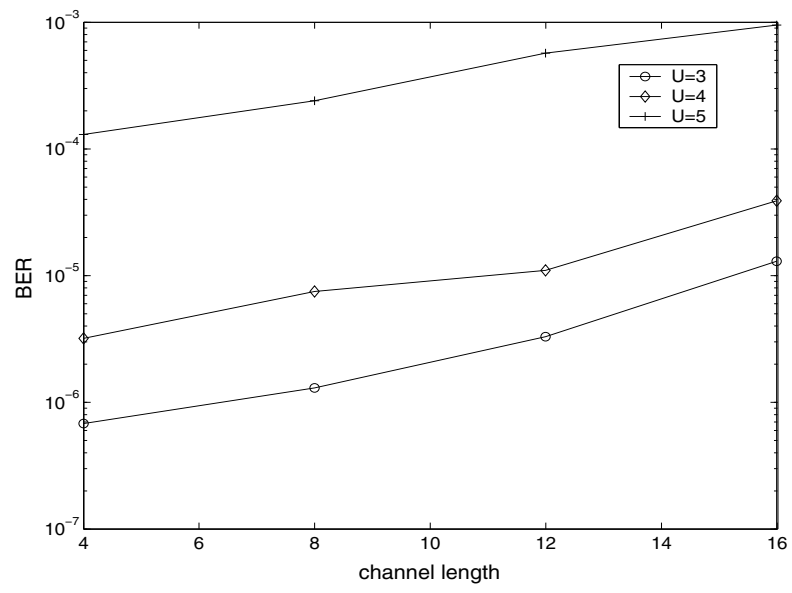

Fig. 9. BER performance with different number of users.

The BER performance is plotted in Fig. 9, and effective throughput is plotted in Fig. 10. Both BER and effective throughput degrade with longer channel length as expected (see Section V-C). More remarkably, the increase in the number of users has a great influence on BER and effective throughput performance. Although BER degrades by almost an order of magnitude when the number of users is increased from 3 to 4 , effective throughput is reduced slightly. However, with 5 users, effective throughput is dramatically reduced: at channel length of 16 , effective throughput even approaches 0 . This is because the antenna freedom is mostly used for interference cancellation so that there is little diversity gain. Therefore, the addition of a new user should be cautioned. In the above example, the number of user should be no more than 4 , although the theoretical limit is 6 with 6 BS antennas.

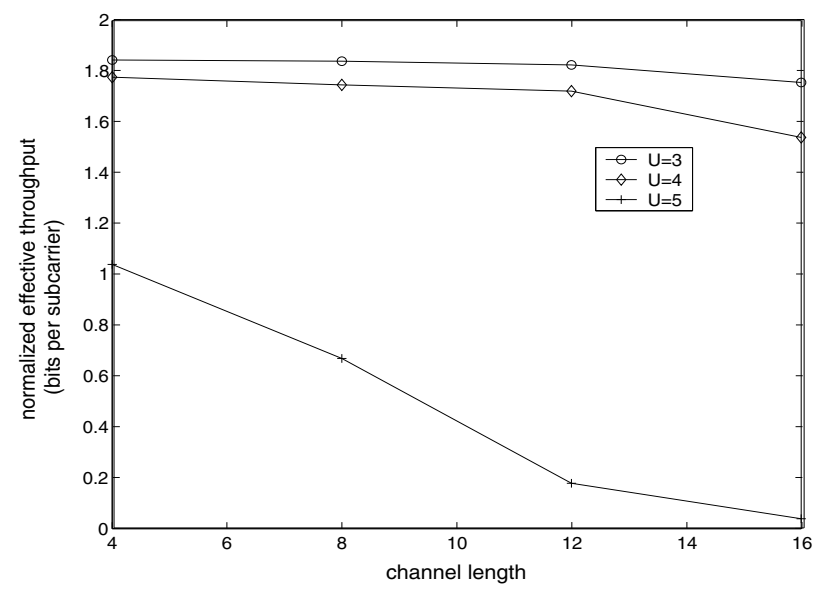

Fig. 10. Normalized effective throughput for different number of users.

\section{F. Influence of SNR}

The BER and effective throughput are plotted against SNR in Fig. 11 and Fig. 12, respectively. In the simulation, we have 3 users, and $\left(M_{f}=8, M_{t}=5\right)$. For a fair comparison, all modulation schemes have the same SNR.

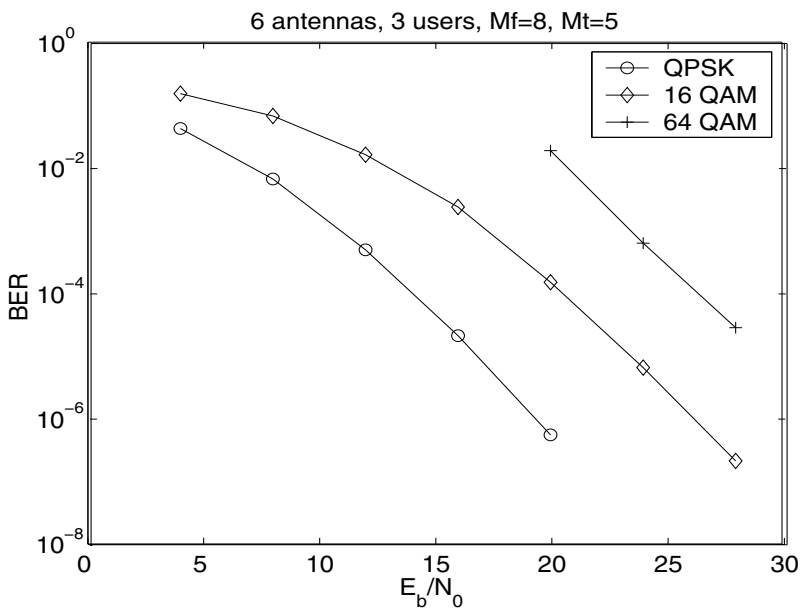

Fig. 11. BER performance with respect to SNR.

The results in Fig. 11 show that 16 QAM and 64 QAM require higher power for good BER performance compared to QPSK. 
To achieve the same BER performance as QPSK, 16 QAM needs about $6 \mathrm{~dB}$ more power, while 64 QAM needs about $12 \mathrm{~dB}$ more. In contrast, there is much less SNR difference to achieve the same effective throughput. From Fig. 12, we observe that 16 QAM needs about $3 \mathrm{~dB}$ more power than QPSK to achieve 1.7 bits/subcarrier, while 64 QAM needs about $9 \mathrm{~dB}$ more. This is because the higher order modulation has more bits on a subcarrier, which compensates for the power requirement for BER.

Note also that the modulation scheme achieving the best effective throughput depends on the value of SNR. For example, when SNR is lower than $16 \mathrm{~dB}$, QPSK is the only option, since 16 QAM and 64 QAM deliver no effective throughput. When SNR is between $20 \mathrm{~dB}$ and $26 \mathrm{~dB}$ (approximately), 16 QAM is the best. It produces effective throughput above 2 bits/subcarrier, while the effective throughput for QPSK saturates at 1.9 bits/subcarrier, and effective throughput for 64 QAM is lower than that of 16 QAM. When SNR is above $26 \mathrm{~dB}$, 64 QAM is the best, because QPSK and 16 QAM saturate at $1.9 \mathrm{bits} /$ subcarrier and $3.7 \mathrm{bits} /$ subcarrier, respectively.

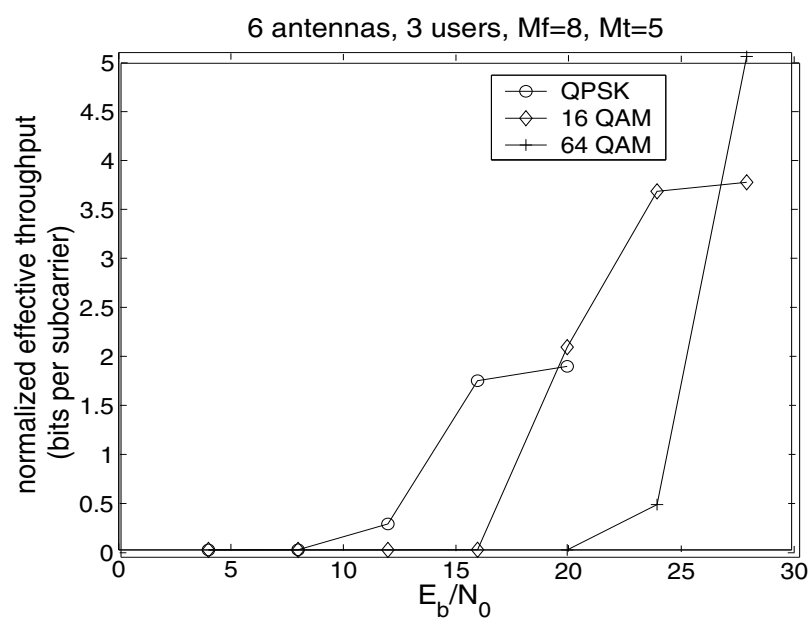

Fig. 12. Normalized effective throughput with respect to SNR.

As observed from Fig. 12, the curve of effective throughput can be characterized by three segments corresponding to two critical SNR turning points. When SNR is below a cut-off value $\gamma_{1}$, effective throughput is almost zero. When SNR is higher than another threshold value $\gamma_{2}$, effective throughput becomes saturated. Between the two values, the effective throughput grows almost linearly with the SNR.

The above phenomena can be explained as follows. When SNR is low (e.g., lower than $\gamma_{1}$ ), $P_{e}$ is close to 1 so that effective throughput is 0 . As a rule of thumb, we can set $\gamma_{1}$ as the SNR for $P_{e}=0.9$. Obviously, a modulation scheme should always operate above $\gamma_{1}$. On the other hand, the normalized effective throughput is expressed in (10) as $\bar{T}_{u}=\left(1-P_{e}\right) N_{d} M_{I} r_{c} / N_{c}$, where $N_{d}$ is the average number of data subcarriers in an OFDM symbol. From our arrangement of pilots, $N_{d}$ can be calculated as

$$
N_{d}=N_{c}-N_{c} / M_{f} / M_{t}
$$

Thus, when SNR is high (e.g., higher than $\gamma_{2}$ ), $P_{e} \rightarrow 0$, so that
$\bar{T}_{u}$ is close to the upper limit

$$
N_{d} M_{I} r_{c} / N_{c}
$$

For this reason, there is no need to operate at SNR above $\gamma_{2}$, since effective throughput will saturate.

The threshold $\gamma_{2}$ can be intuitively determined from the BER as follows. We expect effective throughput to saturate when $P_{e} \leq 0.1$ (or $1-P_{e} \geq 0.9$ ). From (16), $P_{e} \approx L_{d} P_{b}$. With packet length $L_{d}=500$ bytes, or 4000 bits, the corresponding BER is approximately $2.5 \times 10^{-5}$ when $P_{e}=0.1$. Thus, the SNR threshold $\gamma_{2}$ should correspond to a BER of $2.5 \times 10^{-5}$.

\section{G. Summary of Simulation Results}

From our simulation results, we have the following conclusions: 1) Channel delay spread is more influential than Doppler spread on system performance under our experimental settings. Consequently, the pilot spacing in the frequency domain is more important than in the time domain; 2) Caution should be taken when determining the number of operating users, since performance can be seriously degraded when the number of users is close to the number of antennas; 3) For any modulation scheme, the operating SNR should fall between two cutoff SNR values obtained from the effective throughput curve: the effective throughput is almost zero when the SNR is smaller than the lower threshold, and is saturated when higher the upper threshold.

\section{CONCLUSION}

In this paper, effective throughput is proposed as a unified performance measure for wireless OFDM/SDMA systems. This parameter incorporates PER, number of data subcarriers, modulation index and coding rate. System optimization can be achieved through the maximization of effective throughput. Through computer simulations, we have demonstrated how system performance is influenced by various operating parameters. Simulation results have revealed a set of conclusions for system optimization, which are otherwise not obtainable without the aid of effective throughput. Therefore, it is confirmed that effective throughput can serve as a useful performance measure for OFDM/SDMA wireless systems. The impact of channel coding is not considered in this paper, which is to be investigated in future research.

\section{REFERENCES}

[1] T. Keller, L. Hanzo, "Adaptive multicarrier modulation: A convenient framework for time-frequency processing in wireless communications," Proc. IEEE, vol. 88, pp. 611-640, May 2000.

[2] R. van Nee, R. Prasad, OFDM for Wireless Multimedia Communications, Artech House, 2000.

[3] Y. Li, N. R. Sollenberger, "Adaptive antenna arrays for OFDM systems with cochannel interference," IEEE Trans. Commun., vol. 47, no. 2, pp. 217-229, Feb. 1999.

[4] K. K. Wong, R. Cheng, K. B. Letaief, R. D. Murch, "Adaptive antennas at the mobile and base stations in an OFDM/TDMA system," IEEE Trans. on Commun., vol. 49, pp. 195-206, Jan. 2001.

[5] P. Vandenameele, L. Van Der Perre, M. Engels, B. Gyselinckx, H. De Man, "A combined OFDM/SDMA approach," IEEE J. Select. Areas Commun., vol. 18, pp. 2312-2321, Nov. 2000

[6] C. Garnier, M. Loosvelt, V. Le Thuc, Y. Delignon, L. Clavier, "Performance of an OFDM-SDMA based system in a time-varying multi-path channel," Proc. VTC'2001, Fall, 2001, vol. 3, 1686-1690. 
[7] J. Cavers, "An analysis of pilot symbol assisted modulation for Rayleigh fading channels (mobile radio)," IEEE Trans. Veh. Technol., vol. 40, no. 4, pp. 686-693, 1991.

[8] Y. Li, "Pilot-symbol-aided channel estimation for OFDM in wireless systems," IEEE Trans. Veh. Technol., vol. 49, no. 4, pp. 1207-1215, Jul. 2000.

[9] M. Morelli, U. Mengali, "A comparison of pilot-aided channel estimation methods for OFDM systems," IEEE Trans. Signal Processing, vol. 49, no. 12, pp. 3065-3073, Dec. 2001.

[10] M. Garcia, J. Paez-Borrallo, S. Zazo, "DFT-based channel estimation in 2D-pilot-symbol-aided OFDM wireless systems," Proc. VTC'2001, Spring, 2001, vol. 2, 810-814.

[11] L. Hanzo, W. Webb, T. Keller, Single- and Multi-carrier Quadrature Amplitude Modulation: Principles and Applications for Personal Communications, WLANs and Broadcasting, John Wiley \& Sons, 2000.

[12] R. T. Compton, Adaptive Antennas: Concepts and Performance, PrenticeHall, 1988.

[13] R. Negi, J. Cioffi , "Pilot tone selection for channel estimation in a mobile OFDM system," IEEE Trans Consum. Electron., vol. 44, no. 3, pp. 11221128, Aug. 1998.

[14] Wireless LAN Medium Access Control (MAC) and Physical Layer (PHY) specifi cation: High-speed Physical Layer in the $5 \mathrm{GHz}$ Band, IEEE std 802.11a-1999.

[15] Broadband Radio Access Networks (BRAN): High Performance Radio Local Area Networks (HIPERLAN) Type 2, System Overview, ETSI TR $101683, \mathrm{v} 0.1 .2,1999$.

[16] C. Ahn, I. Sasase, "The effects of modulation combination, target BER, Doppler frequency, and adaptation interval on the performance of adaptive OFDM in broadband mobile channel," IEEE Trans. Consum. Electron., vol. 48, no. 1, pp. 167-174, Feb. 2002.

[17] K. Witrisal, Y. Kim, R. Prasad, "A novel approach for performance evaluation of OFDM with error correction coding and interleaving," Proc. VTC'99, Fall 1999, vol. 1, pp. 294-299.

[18] R. Hoshyar, S. Jamali, A. Bahai, "Turbo coding performance in OFDM packet transmission,' Proc. VTC'2000, Spring, 2000, vol. 2, pp. 805-810.

[19] S. Muller-Weinfurtner, "Coding for multiple antenna transmission in fast fading and in OFDM," Proc. ICC'02, 2002, vol. 3, 1601-1605.

[20] K. Joonsuk, L. J. Cimini, J. C. Chuang, "Coding strategies for OFDM with antenna diversity high-bit-rate mobile data applications," Proc. VTC'98, 1998, vol. 2, pp. 763-767.

[21] P. Dent, G. E. Bottomley, T. Croft, "Jakes fading model revisited," Electron. Lett., vol. 29, no. 3, pp. 1162-1163, June 1993. 